\title{
Temperature profiles for germination of big sagebrush seeds from native stands
}

\author{
JAMES A. YOUNG, DEBRA E. PALMQUIST, AND RAYMOND A. EVANS
}

\begin{abstract}
The germination of seeds of big sagebrush (A rtemisia tridentata, Nutt.) from 5 locations on the margin of the Carson Desert, Nevada, was studied over 5 growing seasons. Temperature profiles for germination were developed for seeds collected from each location. The profile consisted of 55 constant or alternating temperatures from 0 through $40^{\circ} \mathrm{C}$. Seed quality was high with optimum germination, defined as germination not different from the maximum observed at the 0.01 level of probability, averaging $80 \%$ for all years and all locations. Differences in germination parameters existed among locations, but not within locations among years of production. Differences among years of production were most pronounced at extreme incubation temperatures. The most frequent temperature regime supporting optimum germination of big sagebrush seeds was $15 / 20^{\circ} \mathrm{C}\left(15^{\circ} \mathrm{C}\right.$ for 16 hours and $20^{\circ} \mathrm{C}$ for 8 hours in each 24-hour cycle).
\end{abstract}

Key Words: range ecology, seedbed temperatures, microenvironments, temperate deserts

Big sagebrush (Artemisia tridentata, Nutt.) is the dominant shrub on millions of hectares of western rangelands in North America (West 1983). The herbage of big sagebrush is an important component of the diet of some native upland birds and in the winter diet of native large herbivores. Big sagebrush is a very competitive species which can suppress the growth of herbaceous forage species. Small changes in the relative dominance of big sagebrush, as indicated by increases in projected cover of the shrub, may severely impact production and density of other herbaceous species (Sneva 1972).

It is relatively easy to control big sagebrush with mechanical or herbicidal treatments (Pechanec et al. 1965). The cost-benefit ratio of these treatments is determined by how soon big sagebrush seedlings become established after the shrub reduction treatment. Big sagebrush does not sprout from crown or root buds after the aerial portion of the shrub is removed. Shrub reestablishment has to be from seeds.

Despite the abundance and importance of big sagebrush, information on the seedling ecology of this species is often fragmentary and sometimes contradictory. The contradictions are often between observations from the field and the results of laboratory experiments. Field observations have suggested there are years when seeds of big sagebrush are more viable, as indicated by successful seedling establishment, than in others (Bleak and Miller 1955). This has not been confirmed by laboratory experiments using seeds collected from the same stand in different years (Harniss and McDonough 1976). In general, the germination of big sagebrush seeds can be characterized as highly variable. Occasionally, seed collections produce high levels of germination, but germination below 50 or even $30 \%$ has been more common (McDonough and Harniss 1974).

Big sagebrush seeds germinate in the dark without pretreatment. Some collections of freshly harvested seeds have been found to have afterripening requirements that must be met before germination will occur. Seeds of the subspecies mountain big sagebrush

\footnotetext{
Authors are range scientist. mathematician/statistician, and retired range scientist, respectively. USDA/ARS, 920 Valley Road, Reno, Nevada 89512.

Manuscript accepted 15 October 1990.
}

[Artemisia tridentata, Nutt. subsp. vaseyana (Rydb.) Beetle] have enhanced germination following cool-moist stratification (McDonough and Harniss 1974).

The seeds of big sagebrush illustrate some basic problems in interpreting results of germination experiments with seeds collected from native stands. Should seeds be collected from more than 1 stand; in more than 1,2 , or $x$ number of years of production?

Our purpose in this study was to develop germination-temperature profiles for collections of big sagebrush seeds from 5 native stands over 5 growing seasons.

\section{Materials and Methods}

Seeds of big sagebrush were collected from 5 native stands located in western Nevada. The stands from which the seeds were collected were located on the lower slopes of mountain ranges that parallel the eastern slope of the Sierra Nevada and border the Carson Desert. The Churchill Canyon sites (Numbers 1, 3, and 5) were located $100 \mathrm{~km}$ southeast and the Granite Peak and Medell Flat sites $40 \mathrm{~km}$ north of Reno, Nevada. The physical environment and structure of the plant communities at each collection site were described in detail in previous publications in this series (Young and Evans 1988, Young et al. 1989). Only environmental parameters that are essential to the interpretation of the results of this study will be reported. The subspecies mountain big sagebrush occurred at Churchill Canyon Number 5 and Granite Peak. Basin big sagebrush ( $A$. tridentata, Nutt. subsp. tridentata, Nutt.) occurred at the other 3 sites. All of the sites represent varying levels of arid extremes for the respective subspecies of big sagebrush.

Seeds were collected at maturity from 1982 through 1986 from the same plants at each location. At least 25 plants composed each population. Identical threshing, cleaning, and storage procedures were used each year (Young and Evans 1988). Immediately after seeds were harvested in December, germination tests were initiated and repeated 12 months later. Seeds were stored in paper bags on the laboratory shelf for any delayed experiments.

In all studies, 4 replications of 25 seeds each were used in a randomized block design. Germination tests were conducted with seeds on top of 1 thickness of germination paper in closed petri dishes. The plates were kept moist with tap water. Tests were conducted in dark germinators for 4 weeks with germination counts at 1,2, and 4 weeks. Seeds were considered germinated when the radicle emerged $1 \mathrm{~mm}$. Incubation temperatures consisted of a constant $0,2,5^{\circ} \mathrm{C}$, and at $5^{\circ} \mathrm{C}$ increments through $40^{\circ}$ C. In addition, alternating regimes of 16 hours at each constant temperature, plus 8 hours at each possible higher temperature, were included. For example, $35^{\circ} \mathrm{C}$ alternated with $40^{\circ} \mathrm{C}$ only, while $0^{\circ} \mathrm{C}$ alternated with $2,5,10,15,20,25,30,35$, and $40^{\circ} \mathrm{C}$. This made a total of 55 constant and alternating temperature regimes.

The germination response of the seeds from the 5 locations for the 5 seed production years was compared using the following seedbed temperature definitions:

a) Very cold: $0 / 0,0 / 2,0 / 5$, and $2 / 2^{\circ} \mathrm{C}$

b) Cold: $0 / 10,0 / 15,2 / 5,2 / 10,2 / 15,5 / 5$, and $5 / 10^{\circ} \mathrm{C}$

c) Cold fluctuating: $0 / 20$ through $0 / 40^{\circ} \mathrm{C}$ and $2 / 20$ through $2 / 40^{\circ} \mathrm{C}$ 
d) Fluctuating: $5 / 30$ through $5 / 40^{\circ} \mathrm{C}, 10 / 35,10 / 40^{\circ} \mathrm{C}$, and $15 / 40^{\circ} \mathrm{C}$

e) Moderate: $5 / 15$ through $5 / 25^{\circ} \mathrm{C}, 10 / 10$ through $10 / 30^{\circ} \mathrm{C}$, $15 / 15$ through $15 / 35^{\circ} \mathrm{C}, 20 / 20$ through $20 / 35^{\circ} \mathrm{C}, 25 / 25^{\circ} \mathrm{C}$, and $25 / 30^{\circ} \mathrm{C}$

f) Warm: $20 / 40,25 / 35$, and $25 / 40^{\circ} \mathrm{C}, 30 / 30$ through $30 / 40^{\circ} \mathrm{C}$, $35 / 35,35 / 40$, and $40 / 40^{\circ} \mathrm{C}$

These categories of seedbed temperatures reflect spring germination conditions, based on several years of microenvironmental monitoring in field seedbeds in the Great Basin big sagebrush zone (Evans et al. 1970; Evans and Young 1970, 1972).

Data from each base temperature and its alternating temperature regimes were used to develop regression equations that in turn were used to generate a quadratic response surface with estimated germination means and confidence intervals at the $1 \%$ level of probability (Evans et al. 1982). Germination profiles were compared using techniques developed by Palmquist et al. (1987).

A number of germination parameters were synthesized from the quadratic response surfaces (Table 1) (Young and Evans 1982). These were used in interpreting the germination-temperature profiles.

\section{Results and Discussion}

To put this study in perspective, the biological setting of the study area needs to be clearly visualized. Big sagebrush characterized $10 \%$ of the vegetation of the area that is now the contiguous 48 states of the United States (West 1983). The 5 locations from which we collected seeds over a 5-year period represent a tiny fraction of this area and an extreme edge of the adaptation of the species. Within this small percentage of the habitat for big sagebrush, we dealt with 2 subspecies of big sagebrush. These subspecies can be recognized by experienced observers in the field by growth form and flower stalk differences, but not on the normal flora characteristics of systematic botany. Because mountain big sagebrush characteristically is found at more mesic, higher elevation sites, the 2 sites, Churchill Canyon No. 5 and Granite Peak, are probably more truly on the extremes of its distribution than the 3 basin big sagebrush sites.

\section{Germination Values}

Mean and maximum observed germination and mean germination for the temperature regimes that supported optimum germina- tion are gross measures of the amount of germination of each source at each site annually. Mean germination for the entire profile can be criticized as too gross an average of germination over optima and sub- or super-optima temperatures. However, if we ignore relative values and deal with variability among years, it proves a useful statistic (Table 1). To do this, we must assume that each seed production year is a discrete product of the numerous environmental factors that influence seed production and quality.

Mean germination for the entire 55 temperature profiles differed significantly $(P \leq 0.01)$ among years for big sagebrush seeds harvested at Granite Peak, Churchill Canyon Number 5, and Medell Flat, but was not markedly different at the other 2 sites (Table 2). There was no clear relation between the average precipitation at a site during a given year (Young and Evans 1988) and the variability in mean germination of the big sagebrush seeds among years. This has to be qualified for the Churchill Canyon Number 1 site where no seeds were produced during the dry year of 1984 . The 2 sites with the most effective moisture for plant growth, Granite Peak and Medell Flat, were among the more variable in mean germination (See Young et al. 1989 for estimates of effective moisture for plant growth). Granite Peak had its highest mean germination in the driest year.

When all the sites were averaged together for mean germination (total of 137,500 observations), there were no significant differences $(P \geq 0.01)$ among years (Table 2), despite the increased level of precision as error terms were pooled. There were significant differences $^{\prime}(P \leq 0.01)$ among locations when the years were averaged (Table 2). The same differences were also apparent annually among sources for mean germination (differences not shown in Table 2 for clarity). The 3 sources of basin big sagebrush seed differed among themselves; they also overlapped statistically and differed significantly ( $P \leq 0.01$ ) from the 2 sources of mountain big sagebrush seed, which also differed from each other.

Maximum germination and mean germination for the optimal temperature regimes produced similar results for the 5 sources of big sagebrush over the 5 years of seed collection (Table 2). The maximum germination parameter provided a measure of the seed populations viability. Within a given site, maximum observed germination was quite variable among years (Table 2). For seeds of big sagebrush collected at Medell Flat, maximum germination ranged from 81 to $100 \%$ for 4 out of the 5 years and dropped to $63 \%$

Table 1. Germination parameters synthesized from quadratic response surfaces (Young and Evans 1982).

\begin{tabular}{|c|c|c|}
\hline \multirow[b]{2}{*}{ Synthesized parameter } & Derived & \multirow[b]{2}{*}{ Purpose } \\
\hline & Synthesized within profiles & \\
\hline Mean germination & $\frac{\Sigma}{55}$ & Gross comparison of profiles \\
\hline Mean of regimes with some germination & $\frac{\Sigma \text { with germination }}{\text { Number regimes with germination }}$ & $\begin{array}{l}\text { Provides measure of breadth of germination } \\
\text { response }\end{array}$ \\
\hline Percentage of regimes with germination & $\frac{\text { Number with germination }}{55}$ & $\begin{array}{l}\text { Indication of breadth of germination responses } \\
\text { in relation to temperature }\end{array}$ \\
\hline \multirow[t]{2}{*}{$\begin{array}{l}\text { Percentage of regimes with optimum } \\
\text { germination }\end{array}$} & $\begin{array}{l}\text { Number of regimes with germination not less than } \\
\text { maximum observed minus confidence interval }\end{array}$ & $\begin{array}{l}\text { Provides indication of breadth of temperatures } \\
\text { that support optimum germination }\end{array}$ \\
\hline & 55 & \\
\hline Mean of optima & $\frac{\Sigma \text { of optima }}{\text { Number or optıma regimes }}$ & $\begin{array}{l}\text { Provides measure of potential germination } \\
\text { of most adapted temperature regimes }\end{array}$ \\
\hline Maximum germination & Highest regime & Indication of potential viability \\
\hline Frequency of optima & $\begin{array}{l}\text { Number of times temperature } \\
\text { regimes supports optima } \\
\text { Total number of tests }\end{array}$ & $\begin{array}{l}\text { Provides an estimate of optimum temperatures } \\
\text { for germination sith statistical precision }\end{array}$ \\
\hline
\end{tabular}


Table 2. Comparison of average, maximum and mean of optima temperature regimes for germination for 5 sources of big sagebrush seeds collected from the same native plants in 5 growing seasons. Germination parameters based on 55 constant or alternating temperatures. Germination test conducted within 60 days of seed harvest. ${ }^{1}$

\begin{tabular}{|c|c|c|c|c|c|c|c|}
\hline \multirow[b]{2}{*}{ Germination parameter } & \multirow[b]{2}{*}{ Location } & \multicolumn{5}{|c|}{ Year seed produced } & \multirow[b]{2}{*}{ Mean } \\
\hline & & 1982 & 1983 & 1984 & 1985 & 1986 & \\
\hline \multirow[t]{2}{*}{ Mean germination } & $\begin{array}{l}\text { Granite Peak } \\
\text { Churchill Canyon } 5 \\
\text { Churchill Canyon } 1 \\
\text { Churchill Canyon } 3 \\
\text { Medell Flat }\end{array}$ & $\begin{array}{l}34 \mathrm{c} \\
48 \mathrm{~b} \\
59 \\
71 \\
51 \mathrm{~b} \\
\end{array}$ & $\begin{array}{l}35 \mathrm{c} \\
46 \mathrm{~b} \\
69 \\
69 \\
59 \mathrm{ab} \\
\end{array}$ & $\begin{array}{l}--(\%) \\
55 \mathrm{a} \\
50 \mathrm{~b} \\
\overline{77}^{2} \\
71 \mathrm{a} \\
\end{array}$ & $\begin{array}{l}50 \mathrm{~b} \\
68 \mathrm{a} \\
62 \\
66 \\
49 \mathrm{~b} \\
\end{array}$ & $\begin{array}{l}42 \mathrm{bc} \\
56 \mathrm{ab} \\
60 \\
72 \\
60 \mathrm{ab} \\
\end{array}$ & $\begin{array}{l}43 \mathrm{z} \\
54 \mathrm{y} \\
63 \mathrm{wx} \\
71 \mathrm{w} \\
58 \mathrm{x}\end{array}$ \\
\hline & Mean & $\overline{53}$ & $\overline{55}$ & $\overline{63}$ & 59 & 58 & \\
\hline \multirow[t]{2}{*}{ Mean germination } & $\begin{array}{l}\text { Granite Peak } \\
\text { Churchill Canyon } 5 \\
\text { Churchill Canyon } 1 \\
\text { Churchill Canyon } 3 \\
\text { Medell Flat }\end{array}$ & $\begin{array}{l}63 \mathrm{c} \\
86 \\
87 \\
95 \\
81 \mathrm{~b} \\
\end{array}$ & $\begin{array}{l}57 \mathrm{c} \\
79 \\
93 \\
95 \\
92 \mathrm{ab} \\
\end{array}$ & $\begin{array}{r}87 a \\
89 \\
-29 \\
99 \\
100 a \\
\end{array}$ & $\begin{array}{l}75 \mathrm{ab} \\
93 \\
90 \\
84 \\
63 \mathrm{c} \\
\end{array}$ & $\begin{array}{l}68 \mathrm{bc} \\
90 \\
90 \\
92 \\
96 \mathrm{a} \\
\end{array}$ & $\begin{array}{l}70 \mathrm{z} \\
87 \mathrm{y} \\
90 \mathrm{y} \\
93 \mathrm{y} \\
86 \mathrm{y}\end{array}$ \\
\hline & Mean & 82 & 83 & 94 & 81 & 87 & \\
\hline \multirow[t]{2}{*}{ Mean of optima } & $\begin{array}{l}\text { Granite Peak } \\
\text { Churchill Canyon } 5 \\
\text { Churchill Canyon } 1 \\
\text { Churchill Canyon } 3 \\
\text { Medell Flat }\end{array}$ & $\begin{array}{l}59 \mathrm{~b} \\
82 \mathrm{ab} \\
84 \\
93 \\
78 \mathrm{~b} \\
\end{array}$ & $\begin{array}{l}55 \mathrm{~b} \\
75 \mathrm{~b} \\
91 \\
93 \\
89 \mathrm{ab} \\
\end{array}$ & $\begin{array}{l}85 \mathrm{a} \\
86 \mathrm{ab} \\
-2^{2} \\
94 \\
99 \mathrm{a} \\
\end{array}$ & $\begin{array}{l}73 \mathrm{a} \\
91 \mathrm{a} \\
88 \\
82 \\
61 \mathrm{c} \\
\end{array}$ & $\begin{array}{l}56 \mathrm{~b} \\
84 \mathrm{ab} \\
90 \\
90 \\
88 \mathrm{ab} \\
\end{array}$ & $\begin{array}{l}66 \mathrm{z} \\
84 \mathrm{y} \\
88 \mathrm{y} \\
90 \mathrm{y} \\
83 \mathrm{y}\end{array}$ \\
\hline & Mean & 79 & 81 & 91 & 79 & 82 & \\
\hline
\end{tabular}

'Means followed by the same letter within location (a through $c$ ) or within overall means for each parameter ( $w$ through $z$ ) are not significantly different at the 0.01 level of probability as determined by Duncan's Multiple Range test. No letters indicate no significant differences to apply. We assumed that seed production years are discrete biologically.

${ }^{2}$ No seed produced at this location this year.

Table 3. Comparison of percentage of temperature regimes with some germination and with optimum germination for 5 sources of big sagebrush seeds collected from the same native plants in 5 growing seasons. Germination parameters based on 55 constant or alternating temperatures. Germination test conducted within 60 days of seed harvest. 1

\begin{tabular}{|c|c|c|c|c|c|c|c|}
\hline \multirow[b]{2}{*}{ Germination parameter } & \multirow[b]{2}{*}{ Location } & \multicolumn{5}{|c|}{ Year seed produced } & \multirow[b]{2}{*}{ Mean } \\
\hline & & 1982 & 1983 & 1984 & 1985 & 1986 & \\
\hline \multirow[t]{2}{*}{$\begin{array}{l}\text { Regimes with some } \\
\text { germination }\end{array}$} & $\begin{array}{l}\text { Granite Peak } \\
\text { Churchill Canyon } 5 \\
\text { Churchill Canyon } 1 \\
\text { Churchill Canyon } 3 \\
\text { Medell Flat }\end{array}$ & $\begin{array}{l}96 \\
95 \\
96 \\
98 \\
96\end{array}$ & $\begin{array}{l}95 \\
91 \\
98 \\
98 \\
98\end{array}$ & $\begin{array}{c}--(\%) \\
98 \\
98 \\
-2 \\
100 \\
100\end{array}$ & $\begin{array}{l}95 \\
98 \\
96 \\
98 \\
98\end{array}$ & $\begin{array}{l}95 \\
95 \\
96 \\
98 \\
98 \\
96\end{array}$ & $\begin{array}{l}96 \\
96 \\
97 \\
98 \\
98\end{array}$ \\
\hline & Mean & 96 & 96 & 99 & 97 & 97 & \\
\hline \multirow[t]{2}{*}{$\begin{array}{l}\text { Regimes with optimum } \\
\text { germination }\end{array}$} & $\begin{array}{l}\text { Granite Peak } \\
\text { Churchill Canyon } 5 \\
\text { Churchill Canyon } 1 \\
\text { Churchill Canyon } 3 \\
\text { Medell Flat }\end{array}$ & $\begin{array}{l}15 \mathrm{~b} \\
13 \\
16 \\
13 \mathrm{~b} \\
8 \mathrm{c} \\
\end{array}$ & $\begin{array}{l}22 \mathrm{a} \\
16 \\
15 \\
16 \mathrm{~b} \\
11 \mathrm{bc}\end{array}$ & $\begin{array}{l}16 \mathrm{~b} \\
13 \\
25 \mathrm{a} \\
15 \mathrm{~b} \\
\end{array}$ & $\begin{array}{l}15 \mathrm{~b} \\
13 \\
16 \\
18 \mathrm{~b} \\
27 \mathrm{a} \\
\end{array}$ & $\begin{array}{l}15 \mathrm{~b} \\
13 \\
15 \\
18 \mathrm{~b} \\
16 \mathrm{~b}\end{array}$ & $\begin{array}{l}17 \\
14 \\
16 \\
18 \\
15\end{array}$ \\
\hline & Mean & 13 & 16 & 17 & 18 & 15 & \\
\hline
\end{tabular}

'Means followed by the same letter are not significantly different at the 0.01 level of probability as determined by Duncan's Multiple Range test. No letters indicate no significant differences to apply. We assume that seed production years are discrete biologically. ${ }^{2}$ No seed produced at this location this year.

in 1985. For the 4 years seed was produced at Churchill Canyon Number 1 , the maximum germination ranged from 87 to $93 \%$ and was not statistically different. Averaging all the sites annually resulted in maximum germination ranging from 82 to $94 \%$, with no significant differences $(P \leq 0.01)$ among years. Averaging years of production together resulted in Granite Peak having a significantly $(P \leq 0.01)$ lower maximum observed germination than the other locations.

Mean germination of the temperature regimes with optimum germination was an interesting parameter for the Churchill Canyon Number 1 and 3 sites because of the constancy among years. These were the 2 driest of the 5 locations where seed was collected, but germination at the optima temperature was always above $82 \%$. This is highly viable for seeds produced under wildland conditions. Equally interesting was the variability observed among years at the other 3 sites for germination at optimal temperatures. At Granite Peak, the range was from 55 to $85 \%$ and at Medell Flat, 61 to $99 \%$. When all the locations were averaged, there were no significant differences $(P \geq 0.01)$ among years.

\section{Breadth of Germination Response}

The germination parameters for temperature regimes with some germination and regimes with optimum germination indicate the breadth of germination responses to incubation temperatures. There were no significant differences among years at a given location or among sites for the percentage of the 55 constant or alternating temperature regimes that supported some germination of big sagebrush seeds (Table 3). At least 52 of the 55 temperatures 
Table 4. Germination of 5 sources of seeds of hig sagebrush collected from the same plants over 5 productive years in relation to 6 categories of seedbed temperatures commonly found in the Intermountain area during the spring germination period. ${ }^{1}$

\begin{tabular}{|c|c|c|c|c|c|c|c|}
\hline \multirow{2}{*}{$\begin{array}{l}\text { Category of } \\
\text { seedbed temperatures }\end{array}$} & \multirow[b]{2}{*}{ Location } & \multicolumn{5}{|c|}{ Year seed produced } & \multirow[b]{2}{*}{ Mean } \\
\hline & & 1982 & 1983 & 1984 & 1985 & 1986 & \\
\hline \multirow[t]{2}{*}{ Very cold } & $\begin{array}{l}\text { Granite Peak } \\
\text { Churchill Canyon } 5 \\
\text { Churchill Canyon } 1 \\
\text { Churchill Canyon } 3 \\
\text { Medell Flat }\end{array}$ & $\begin{array}{l}29 \mathrm{~b}-\mathrm{f} \\
15 \mathrm{def} \\
29 \mathrm{c}-\mathrm{f} \\
38 \mathrm{bc} \\
35 \mathrm{~b}-\mathrm{f} \\
\end{array}$ & $\begin{array}{l}34 \mathrm{~b}-\mathrm{f} \\
36 \mathrm{a}-\mathrm{f} \\
32 \mathrm{c}-\mathrm{f} \\
38 \mathrm{a}-\mathrm{f} \\
15 \mathrm{f} \\
\end{array}$ & $\begin{array}{l}--(\%) \\
25 \mathrm{c}-\mathrm{f} \\
14 \mathrm{ef} \\
-2 \\
46 \mathrm{a}-\mathrm{d} \\
39 \mathrm{~b}-\mathrm{e} \\
\end{array}$ & $\begin{array}{l}-\overline{---} \\
26 \mathrm{c-f} \\
54 \mathrm{ab} \\
41 \mathrm{add} \\
61 \mathrm{a} \\
54 \mathrm{ab} \\
\end{array}$ & $\begin{array}{l}24 \mathrm{c}-\mathrm{f} \\
14 \mathrm{ef} \\
33 \mathrm{c-f} \\
34 \mathrm{~b}-\mathrm{f} \\
29 \mathrm{~b}-\mathrm{f} \\
\end{array}$ & $\begin{array}{l}28 \mathrm{z} \\
27 \mathrm{z} \\
27 \mathrm{z} \\
43 \mathrm{y} \\
39 \mathrm{yz}\end{array}$ \\
\hline & Mean & $29 \mathrm{z}$ & $31 \mathrm{z}$ & 312 & $47 y$ & $27 \mathrm{z}$ & \\
\hline \multirow[t]{2}{*}{ Cold } & $\begin{array}{l}\text { Granite Peak } \\
\text { Churchill Canyon } 5 \\
\text { Churchill Canyon } 1 \\
\text { Churchill Canyon } 3 \\
\text { Medell Flat }\end{array}$ & $\begin{array}{l}42 \mathrm{c-f} \\
39 \mathrm{ef} \\
51 \mathrm{b-f} \\
66 \mathrm{abc} \\
61 \mathrm{a-e} \\
\end{array}$ & $\begin{array}{l}50 \mathrm{c}-f \\
63 \mathrm{a}-\mathrm{e} \\
59 \mathrm{a}-\mathrm{e} \\
59 \mathrm{a}-\mathrm{e} \\
45 \mathrm{def} \\
\end{array}$ & $\begin{array}{l}56 \text { ef } \\
36 \mathrm{f} \\
\frac{-2}{66 \mathrm{a}-\mathrm{d}} \\
68 \mathrm{abc}\end{array}$ & $\begin{array}{l}48 \mathrm{c}-\mathrm{f} \\
75 \mathrm{a} \\
69 \mathrm{ab} \\
74 \mathrm{a} \\
59 \mathrm{a}-\mathrm{e} \\
\end{array}$ & $\begin{array}{l}51 \mathrm{~b}-\mathrm{f} \\
42 \mathrm{c-f} \\
50 \mathrm{c}-\mathrm{f} \\
59 \mathrm{a}-\mathrm{e} \\
63 \mathrm{a}-\mathrm{e} \\
\end{array}$ & $\begin{array}{l}49 \mathrm{z} \\
51 \mathrm{z} \\
57 \mathrm{z} \\
65 \mathrm{y} \\
59 \mathrm{yz}\end{array}$ \\
\hline & Mean & 52 & 55 & 57 & 65 & 53 & \\
\hline \multirow[t]{2}{*}{ Cold fluctuating } & $\begin{array}{l}\text { Granite Peak } \\
\text { Churchill Canyon } 5 \\
\text { Churchill Canyon } 1 \\
\text { Churchill Canyon } 3 \\
\text { Medell Flat }\end{array}$ & $\begin{array}{l}25 \mathrm{fg} \\
22 \mathrm{~g} \\
78 \mathrm{a} \\
80 \mathrm{a} \\
48 \mathrm{def} \\
\end{array}$ & $\begin{array}{l}32 \mathrm{efg} \\
43 \mathrm{~d}-\mathrm{g} \\
73 \mathrm{ab} \\
70 \mathrm{abc} \\
50 \mathrm{bc} \\
\end{array}$ & $\begin{array}{l}25 \mathrm{fg} \\
30 \mathrm{efg} \\
47 \mathrm{c} \\
67 \mathrm{abc}\end{array}$ & $\begin{array}{l}43 \mathrm{c}-\mathrm{f} \\
71 \mathrm{abc} \\
74 \mathrm{a} \\
68 \mathrm{abc} \\
51 \mathrm{~b}-\mathrm{e} \\
\end{array}$ & $\begin{array}{l}28 \mathrm{efg} \\
36 \mathrm{~d}-\mathrm{g} \\
68 \mathrm{abc} \\
74 \mathrm{a} \\
56 \mathrm{bcd}\end{array}$ & $\begin{array}{l}32 \mathrm{z} \\
40 \mathrm{z} \\
59 \mathrm{xy} \\
68 \mathrm{x} \\
54 \mathrm{y}\end{array}$ \\
\hline & Mean & $51 \mathrm{y}$ & $54 \mathrm{y}$ & $34 \mathrm{z}$ & $62 \mathrm{y}$ & $52 \mathrm{y}$ & \\
\hline \multirow[t]{2}{*}{ Fluctuating } & $\begin{array}{l}\text { Granite Peak } \\
\text { Churchill Canyon } 5 \\
\text { Churchill Canyon } 1 \\
\text { Churchill Canyon } 3 \\
\text { Medell Flat }\end{array}$ & $\begin{array}{l}26 \mathrm{fg} \\
28 \mathrm{fg} \\
54 \mathrm{a}-\mathrm{e} \\
76 \mathrm{ab} \\
42 \mathrm{efg} \\
\end{array}$ & $\begin{array}{l}27 \mathrm{fg} \\
28 \mathrm{fg} \\
82 \mathrm{a} \\
81 \mathrm{a} \\
44 \mathrm{efg} \\
\end{array}$ & $\begin{array}{l}25 \mathrm{~g} \\
57 \mathrm{~b}-\mathrm{e} \\
-2 \\
36 \mathrm{~d}-\mathrm{g} \\
43 \mathrm{fg} \\
\end{array}$ & $\begin{array}{l}58 \mathrm{~b}-\mathrm{e} \\
70 \mathrm{a}-\mathrm{d} \\
74 \mathrm{ab} \\
71 \mathrm{abc} \\
51 \mathrm{cde} \\
\end{array}$ & $\begin{array}{l}28 \mathrm{fg} \\
48 \mathrm{def} \\
68 \mathrm{a}-\mathrm{d} \\
68 \mathrm{a}-\mathrm{d} \\
48 \mathrm{def} \\
\end{array}$ & $\begin{array}{l}33 \mathrm{z} \\
46 \mathrm{y} \\
72 \mathrm{x} \\
66 \mathrm{x} \\
46 \mathrm{y}\end{array}$ \\
\hline & Mean & $47 z$ & $52 \mathrm{z}$ & $40 z$ & $65 \mathrm{y}$ & $52 \mathrm{z}$ & \\
\hline \multirow[t]{2}{*}{ Warmer } & $\begin{array}{l}\text { Granite Peak } \\
\text { Churchill Canyon } 5 \\
\text { Churchill Canyon } 1 \\
\text { Churchill Canyon } 3 \\
\text { Medell Flat }\end{array}$ & $\begin{array}{l}13 \text { ef } \\
26 \mathrm{~b}-\mathrm{f} \\
30 \mathrm{~b}-\mathrm{f} \\
41 \mathrm{a}-\mathrm{d} \\
23 \mathrm{def} \\
\end{array}$ & $\begin{array}{l}7 \mathrm{f} \\
28 \text { ef } \\
42 \mathrm{a}-\mathrm{d} \\
46 \mathrm{abc} \\
35 \mathrm{~b}-\mathrm{e} \\
\end{array}$ & $\begin{array}{l}33 \mathrm{a-f} \\
36 \mathrm{a}-\mathrm{e} \\
-2.2 \\
57 \mathrm{ab} \\
58 \mathrm{a} \\
\end{array}$ & $\begin{array}{l}25 \mathrm{c}-\mathrm{f} \\
31 \mathrm{~b}-\mathrm{f} \\
33 \mathrm{bc} \\
29 \mathrm{c-f} \\
24 \mathrm{def} \\
\end{array}$ & $\begin{array}{l}23 \mathrm{def} \\
29 \mathrm{c-f} \\
33 \mathrm{a-f} \\
36 \mathrm{a}-\mathrm{e} \\
25 \mathrm{c}-\mathrm{f} \\
\end{array}$ & $\begin{array}{l}20 \mathrm{z} \\
30 \mathrm{yz} \\
35 \mathrm{y} \\
42 \mathrm{y} \\
36 \mathrm{y}\end{array}$ \\
\hline & Mean & $27 \mathrm{z}$ & $32 \mathrm{z}$ & $46 y$ & $28 \mathrm{z}$ & $29 \mathrm{z}$ & \\
\hline \multirow[t]{2}{*}{ Moderate } & $\begin{array}{l}\text { Granite Peak } \\
\text { Churchill Canyon } 5 \\
\text { Churchill Canyon } 1 \\
\text { Churchill Canyon } 3 \\
\text { Medell Flat }\end{array}$ & $\begin{array}{l}51 \mathrm{cde} \\
74 \mathrm{ace} \\
79 \mathrm{ab} \\
87 \mathrm{ab} \\
69 \mathrm{bcd} \\
\end{array}$ & $\begin{array}{l}45 \mathrm{e} \\
65 \mathrm{~b}-\mathrm{e} \\
87 \mathrm{ab} \\
86 \mathrm{ab} \\
82 \mathrm{ab} \\
\end{array}$ & $\begin{array}{l}67 \mathrm{abc} \\
77 \mathrm{abc} \\
-2.2 \\
91 \mathrm{ab} \\
91 \mathrm{a} \\
\end{array}$ & $\begin{array}{l}68 \mathrm{a}-\mathrm{e} \\
84 \mathrm{ab} \\
78 \mathrm{ab} \\
75 \mathrm{ab} \\
55 \mathrm{de} \\
\end{array}$ & $\begin{array}{l}65 \mathrm{~b}-\mathrm{c} \\
78 \mathrm{ab} \\
84 \mathrm{ab} \\
82 \mathrm{ab} \\
68 \mathrm{abc} \\
\end{array}$ & $\begin{array}{l}59 \mathrm{z} \\
76 \mathrm{y} \\
82 \mathrm{y} \\
84 \mathrm{y} \\
73 \mathrm{y}\end{array}$ \\
\hline & Mean & 72 & 73 & 82 & 72 & 75 & \\
\hline
\end{tabular}

I Within each category of seedbed temperatures means followed by the same letter within location (a through c) are not significantly different at the 0.01 level of probability as determined by regression surface technique with confidence intervals on the means. The overall means for sites and years for each category are compared separately. Means for years horizontally or locations vertically followed by the same letter ( $x$ through $z$ ) are not significantly different at the 0.01 level of probability as determined by Duncan's Multiple Range test. No letters indicate no significant differences.

${ }^{2}$ No seed produced at this location this year.

tested supported some germination. The temperature regimes that usually failed to show any germination were $0 / 40,35 / 40$, and $40 / 40^{\circ} \mathrm{C}$.

The percentage of temperature regimes that support optimum germination is a statistic that must be interpreted with care through comparison with the mean germination of the optima. A low number of optima supporting regimes with high average germination may indicate a highly specific optimum range of temperatures for germination (e.g., Churchill Canyon Number 5 in 1985, Tables 2 and 3). A wide range of optima with high mean germination indicates a lack of a specific optimal temperature for germination (e.g., Churchill Canyon Number 3 in 1984, Tables 2 and 3).

A wide range of optima coupled with a low average germination for the optima temperatures indicates an imprecise, highly variable test (e.g., Medell Flat in 1985, Tables 2 and 3). At some locations there were significant differences $(P \leq 0.01)$ among years. When all locations were averaged together, there were no differences among years.

\section{Optimum Temperature for Germination}

The concept of frequency of optimum temperatures for germination is potentially very useful, but on past experience (e.g., Young and Evans 1982) indicates that it is difficult to explain. The concept is based on multiple germination profiles over time on the same type of seeds. In this study, we had 5 trials for each of 5 locations. From the 25 profiles, only $15 / 20^{\circ} \mathrm{C}$ was an optimum temperature regime in every test (Fig. 1). The regimes $15 / 25,10 / 20$, and $10 / 25^{\circ}$ $C$ were always optimum for at least 1 source, but not for all sources all of the time. A very wide range of temperatures were optimum for germination at least in 1 test. They ranged from 0 to $20^{\circ} \mathrm{C}$ cold-period temperature, alternating with 15 to $35^{\circ} \mathrm{C}$ warm period temperatures. There were no differences between the subspecies, mountain and basin big sagebrush in optima for germination. The $100 \%$ frequency regimes for both subspecies were the core temperatures $15 / 20,15 / 25,10 / 20$, and $10 / 25^{\circ} \mathrm{C}$ temperatures. 


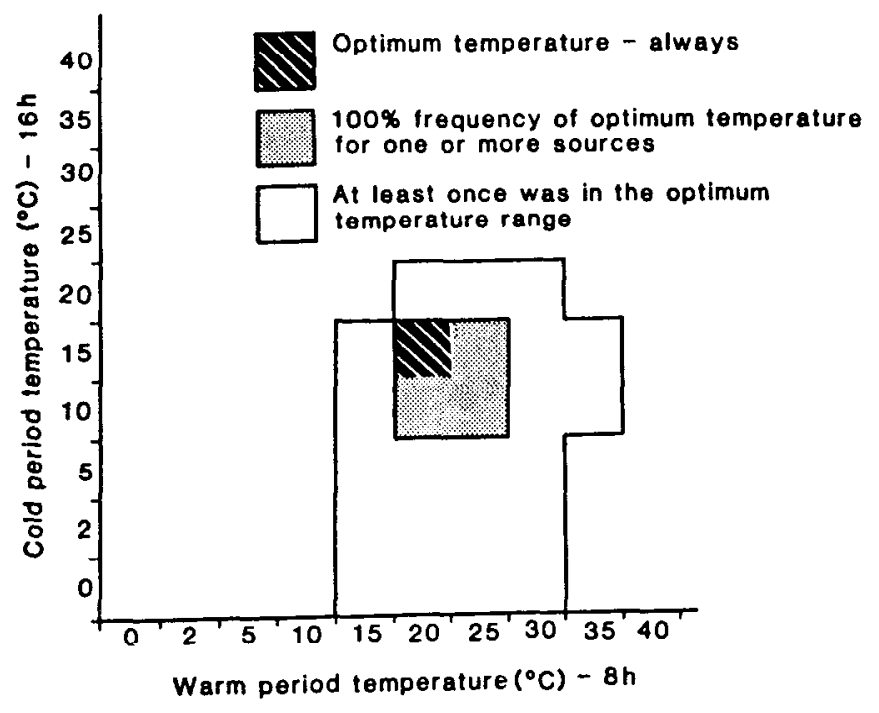

Fig. 1. Frequency of optimum temperature regimes for germination of seeds of big sagebrush.

\section{Delayed Germination}

Germination profiles were conducted within 60 days of harvest and repeated 1 year later. We did not have marked increases in germination in the delayed test which would indicate afterripening requirements (data not shown).

\section{Germination in Relation to Seedbed Temperatures}

Segregating the 55 temperature regime profiles into categories of seedbed temperatures is based on microenvironmental monitoring in the field and provides another means of assessing differences among the collections of big sagebrush seed over time. Germination at very cold temperatures is particularly important in the big sagebrush zone of the Great Basin because of the need to germinate and establish seedings in the spring before the summer drought (Evans et al. 1970). Early spring germination for seeds of big sagebrush is virtually certain, due to the late fall, early winter maturity of the seeds (Young and Evans 1988). Temperatures in this category range from a low of $0 / 0^{\circ} \mathrm{C}$ to a high of $0 / 5^{\circ} \mathrm{C}$. The big sagebrush collections tested in this study are unique in averaging better than $25 \%$ germination at these very cold temperatures (Table 4). Among sources, the Churchill Canyon Number 3 and Medell Flat sources had the highest 5-year average germination at the very cold category of temperature. This represented significant difference $(P \leq 0.01)$ among years, with seeds produced in 1985 having the highest overall mean germination at very cold temperatures, but at each location yearly variation tended to follow different patterns. At Granite Peak, there were no significant differences among years of production in germination at very cold temperatures. Germination of seeds collected at Churchill Canyon Number 3 in this category of seedbed temperatures was always above $30 \%$ and jumped over $60 \%$ with seeds produced in 1985.

The cold seedbed temperature regimes had very cold temperatures alternating with warm temperatures as high as $15^{\circ} \mathrm{C}$. The locations statistically ranked the same as for very cold temperatures, but average germination increased by $20 \%$ (Table 4 ). There were no significant differences among years for overall means, but there were differences in germination among years for specific locations.

Cold fluctuating temperatures are important for the very small seeds of big sagebrush which apparently must germinate on the soil surface. This category of seedbed temperatures contained very cold $\left(0\right.$ or $\left.2^{\circ} \mathrm{C}\right)$, cold-period temperatures with 18 to $40^{\circ} \mathrm{C}$ diurnal fluctuations. Among locations in overall average germination, the 2 locations with mountain big sagebrush were significantly $(P \leq 0.01)$ lower than the 3 basin big sagebrush sites (Table 4). Among years, 1984 had the driest production season (Young and Evans 1988) and was significantly $(P \leq 0.01)$ lower in germination than the other 4 years.

The category of fluctuating temperature contained a $25^{\circ} \mathrm{C}$ diurnal temperature shift with warm period temperatures in the 30 to $40^{\circ} \mathrm{C}$ range. Among locations, the range in germination response extended to $39 \%$ between the low overall average (Granite Peak) and the high (Churchill Canyon Number 1).

Warm temperature regimes had at least $25^{\circ} \mathrm{C}$ minimum temperatures alternating with 30 through $40^{\circ} \mathrm{C}$ warm-period temperatures. These temperatures were not a good environment for germination of big sagebrush seeds, with overall germination dropping to $33 \%$ (Table 4). The highest overall germination among years occurred in 1984, which was the driest growing season (Table 4).

The remainder of the temperature regimes $(35 \%)$ fell in the moderate category of seedbed temperatures. Overall average germination increased to $75 \%$, with only Granite Peak having significantly $(P \leq 0.01)$ lower germination than the other locations (Table 4). For the overall means, there were no differences among years in germination.

The big sagebrush seed in this study was highly viable with the mean germination, at the optimum temperature regime, exceeding $80 \%$, with a range for locations from 66 to $90 \%$. For seeds of a native plant, this is excellent germination. Maximum germination averaged $85 \%$ with a $100 \%$ maximum occurring at 1 location. Payne (1957) obtained a maximum germination of $52 \%$ for big sagebrush seeds from a wide diversity of geographical areas. Mountain big sagebrush seeds have been noted for afterripening requirements and high dormant seeds (McDonough 1974). Seeds of mountain big sagebrush from stands in Idaho have much more severe dormancy controls than those of this study (Harniss and McDonough 1976, McDonough and Harniss 1974). For many of the germination characteristics investigated in this study, the seeds of mountain and basin big sagebrush represent extremes of physiological characteristics, but statistical overlap often blurred distinctions between the populations of the 2 subspecies.

Differences in germination characteristics among locations remained distinct over time while differences among production years became less distinct as sample size (repeated years of production) increased, despite increased levels of precision as the sample size grew. Differences among years of seed production occurred more frequently at exteme versus moderate incubation temperatures. Apparently the populations from which we sampled produced seeds with markedly different germination characteristics for extreme seedbed environments, but not for moderate seedbed environments. This would explain the differences between the experimental results of Harniss and McDonough (1976) and the field observations of Bleak and Miller (1955) or Frischknecht and Bleak (1957).

Based on the plant material used in this study, the ideal single temperature regime for the germination of big sagebrush would be an alternating $15 / 20^{\circ} \mathrm{C}$. This is a relatively warm, late spring temperature regime for the Great Basin (Evans et al. 1970).

\section{Literature Cited}

Bleak, A.T., and W. Miller. 1955. Sagebrush seedling production related to time of mechanical eradication. J. Range Manage. 8:66-69.

Evans, R.A., D.A. Easi, D.N. Book, and J.A. Young. 1982. Quadratic response surface analysis of seed-germination trials. Weed Sci. 30:411-416. 
Evans, R.A., H.R. Holbo, R.E. Eckert, Jr., and J.A. Young. 1970. Functional environment of downy brome communities in relation to weed control and revegetation. Weed Sci. 18:154-162.

Evans, R.A., and J.A. Young. 1970. Plant litter and establishment of alien annual species in rangeland communities. Weed Sci. 18:697-703.

Evans, R.A., and J.A. Young. 1972. Microsite requirements for establishment of alien annual species in rangeland communities. Weed Sci. 20:350-356.

Frischknecht, N.C., and A.T. Bleak. 1957. Encroachment of big sagebrush on seeded range in northeastern Nevada. J. Range Manage. 10:165-170.

Harniss, R.O., and W.T. MeDonough. 1976. Yearly variation in germination in three subspecies of big sagebrush. J. Range Manage. 29:167-168.

McDonough, W.T. 1974. Seed dormancy in Artemisia tridentata Nutt. subspeces vaseyana Rydb. Northwest Sci. 45:17-20.

McDonough, W.T., and R.O. Harniss. 1974. Effect of temperature on germination of three subspecies of big sagebrush. J. Range Manage. 27:204-205.
Palmquist, D.E., R.A. Evans, and J.A. Young. 1987. Comparative analysis of temperature-germination response surfaces. pp. 97-104 in G.W. Frasier and R.A. Evans. (ed). Proc. Symp. Seed and Seedbed Ecology of Rangeland Plants. USDA, ARS. Gov. Printing Off. Washington, D.C.

Payne, G.F. 1957. Some germination studies of Artemisia tridentata. Montana Acad. Sci. Proc. 17:41-42.

Pechanec, J.F., P.A. Plummer, J.H. Robertson, and A.C. Hull. Jr. 1965. Sagebrush control on rangelands. USDA, Agr. Handb. 277.

Sneva, F.A. 1972. Grazing return following sagebrush control in eastern Oregon. J. Range Manage. 25:174-178.

West, N.E. 1983. Temperate deserts and semi-deserts. Ecosystems of the World 5. Elsevier Scientific Publ. Co., Amsterdam.

Young, J.A., and R.A. Evans. 1982. Temperature profiles for germination of cool season range grasses. ARR-W-27. USDA, ARS, Oakland, Calif.

Young, J.A., and R.A. Evans. 1988. Dispersal and germination of big sagebrush (Artemisia tridentata) seeds. Weed Sci. 37:201-206.

Young, J.A., R.A. Evans, and D.E. Palmquist. 1989. Big sagebrush (Arte-

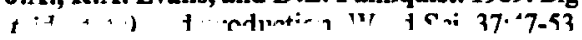

